\title{
Prediction of Alzheimer's Disease in Patients using Features of Pupil Light Reflex to Chromatic Stimuli
}

\author{
Wioletta Nowak* ${ }^{*}$ Minoru Nakayama ${ }^{\dagger}$, Tomasz Kręcicki ${ }^{\ddagger}$ and Andrzej Hachoł* \\ * Institute of Biomedical Engineering and Instrumentation \\ Wrocław University of Science and Technology, Wrocław, Poland 50-370 \\ Email: wioletta.nowak@pwr.edu.pl \\ $\dagger$ Department of Information and Communications Engineering \\ Tokyo Institute of Technology, Tokyo, Japan 152-8552 \\ Email: nakayama@ict.e.titech.ac.jp \\ $\ddagger$ Wrocław Medical University Rektorat, wybrzeze Ludwika Pasteura 1, 50-367 Wrocław, Poland
}

\begin{abstract}
A diagnostic procedure to predict the probability of diagnosing a patient with Alzheimer's Disease (AD) was developed using features of pupil light reflex (PLR) waveforms. 15 features of PLRs for three colours of light pulses at two levels of brightness were measured. Participants were 12 AD patients and 7 control group subjects. A logistic regression analysis was introduced to identify AD patients using two factor scores of features of PLR. The prediction performance of combinations of factor scores for features of PLRs were then evaluated using a test of fitness. An MCMC technique was introduced to estimate the parameters of the regression functions. The model provides a distribution of the probability of diagnosis of $\mathrm{AD}$ patients and control group subjects.
\end{abstract}

\section{INTRODUCTION}

The pupil light reflex (PLR), which produces changes in pupil diameter in response to a light pulse of white or red, has been introduced to diagnose Alzheimer's Disease (AD) [1], [2]. In addition to this, the recent discovery of intrinsically photosensitive retinal ganglion cells (ipRGCs) [3] reveals the possibility of using various diagnostic procedures that involve a shorter light wavelength, such as blue light [4]. For example, PLRs related to ipRGCs can be used to detect symptoms of Age-Related Macular Degeneration (AMD) [5]. Some critical studies have suggested that common sources may be the origin of both AD and AMD diseases [6], [7], [8], [9]. Also, because most AD patients are elderly, the influence of aging on PLRs should be evaluated carefully.

The authors have been studying a diagnostic procedure for detecting AD symptoms using PLRs of various types of light pulses and observing the conditions the light pulses produce [10]. Though these results show the possibility of aiding the diagnosis of the disease, a more flexible procedure is required. In particular, the number of $\mathrm{AD}$ patients used in the experimental survey was restricted, and the assessment of the prediction of accuracy was insufficient. Therefore, significant features of PLRs, which can be used in the diagnostic procedure, should be extracted as necessary. If the distribution of features can be estimated, the possibility of diagnosing AD patients may be predicted using the Bayesian process.

In this paper, features of PLRs are extracted and the differences between $\mathrm{AD}$ patients and control group subjects are discussed. Some procedures for predicting the probability of diagnosing $\mathrm{AD}$ patients are compared. The following topics are addressed:

1) Features of PLRs are analysed and their factors are extracted. The differences in features and factor scores between $\mathrm{AD}$ patients and control group subjects are compared. Also, the influence of aging is evaluated.

2) Logistic regression is introduced to calculate the probability of diagnosing the disease in $\mathrm{AD}$ patients and control group subjects. The models of fitness of the groups are then compared.

3) The MCMC technique is introduced to estimate the parameters of the models, and the performance of the models is discussed.

\section{METHOD}

\section{A. Participants}

A conventional PLR experiment was performed on 19 participants (42 89 years old, mean age:70.6), 12 of which were healthy individuals with normal vision (Control group: 62 89 years old, mean age:72.1) and 7 of which were patients with Alzheimer's Disease (AD Patients: 42 84 years old, mean age:68.1) who had already been diagnosed by medical doctors. It was not easy to invite volunteers who were aged over 80 . The age levels are summarised in Table I.

Informed consent was obtained from all participants prior to the experiment.

\section{B. PLR measurement}

The stimuli consisted of three chromatic lights, red $(635 \mathrm{~nm})$, blue (470nm) and white (CIE x:0.28, y:0.31), at two levels of

TABLE I

NUMBER OF SUBJECTS BY AGE

\begin{tabular}{|l|l|c|c|}
\hline Label & Age & Control & Patient \\
\hline $\mathrm{L}$ & $\leq 70$ & 5 & 3 \\
$\mathrm{M}$ & $71 \sim 80$ & 5 & 3 \\
$\mathrm{H}$ & $81 \leq$ & 2 & 1 \\
\hline
\end{tabular}



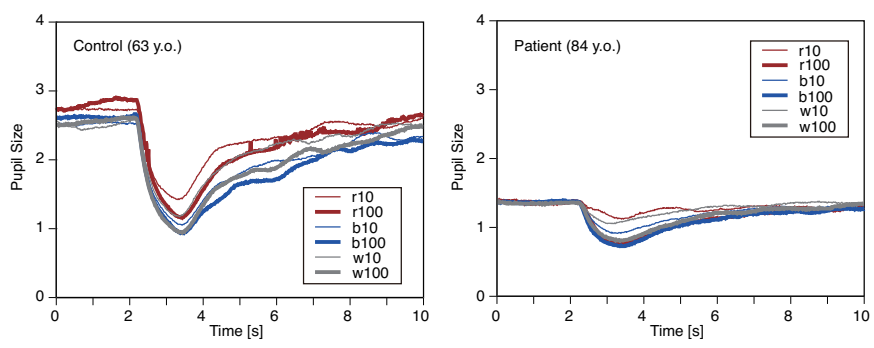

Fig. 1. An example of PLRs for a control group subject and an AD patient

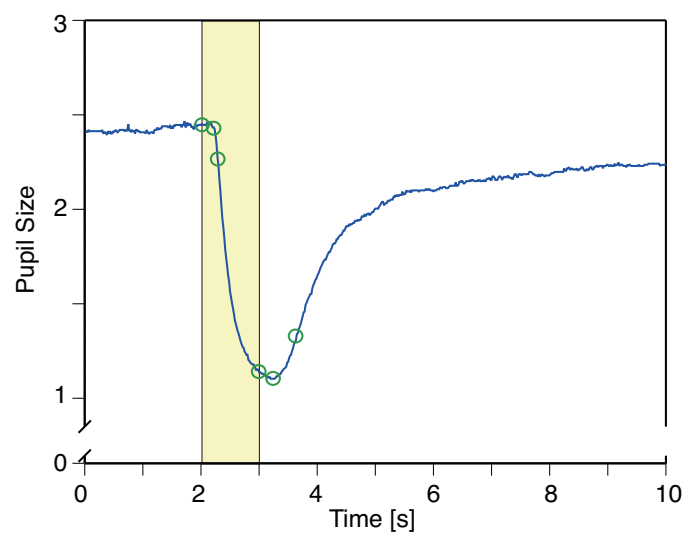

Fig. 2. Feature extraction from PLR responses

brightness $\left(10\right.$ and $\left.100 \mathrm{~cd} / \mathrm{m}^{2}\right)$. These stimuli were labelled as r10, r100, b10, b100, w10 and w100.

The duration of observations was 10 seconds, with the first $2 \mathrm{~s}$ being a pre-stimulus phase as a rest period, followed by a 1s light pulse and $7 \mathrm{~s}$ as a restoration phase. Pupil diameters were measured in $\mathrm{mm}$ at $60 \mathrm{~Hz}$ using a system developed by some of the authors [11]. PLRs for each stimuli were observed in single trials using a repeated-measure design.

Examples of measurements for a healthy individual and for an $\mathrm{AD}$ patient are shown in Figure 1. In these figures, PLRs are illustrated in response to 6 stimuli, namely the 3 colours and two levels of brightness.

\section{RESULTS}

1) Feature definitions: A typical PLR waveform shape is illustrated in Figure 2. In the figure, the light pulse overlaps for a period of $2 \sim 3$ seconds. As the figure illustrates, there are pupillary response delays due to the shrinking of pupil and its restoration to normal size.

Some features are extracted to specify the PLR response, and these variable features are summarised in Table II. They are pupil size, velocity of pupillary change, duration of change, and integration of the waveform. These features are calculated for each PLR response.

\section{A. Comparison of features}

The extracted features for each stimulus were compared between two groups. The results are summarised in Table III.
TABLE II

DEFINITIONS OF PLR FEATURES

\begin{tabular}{|c|l|}
\hline Variable & Definition \& notes \\
\hline ps_base & Mean of the pupil size for time before light pulse \\
ps_lon & Pupil size where light pulse on \\
ps_loff & Pupil size where light pulse off \\
ps_min & Minimum pupil size \\
RA & Range of pupil size (ps_lon - ps_min) \\
\hline v_con & Max amplitude of pupil construction velocity \\
v_rest & Max amplitude of pupil re-constriction velocity \\
ac_max & Min amplitude of pupil acceleration \\
\hline t_delay & Pupil response delay \\
t_min & Time when "ps_min" appears \\
t_v_con & Time when "v_con" appears \\
t_v_rest & Time when "v_rest" appears \\
\hline int_con & Integration of constriction phase \\
int_rest & Integration of restoration phase \\
int & Overall integration (int_con + int_rest) \\
\hline
\end{tabular}

When there is a significant difference between pairs of values, the values are displayed in bold face. As the table shows, there are many significant pairs for the b100 and $\mathrm{r} 10$ conditions, but few pairs for the white stimulus. In regards to the significant differences for the b100 condition, such as pupil size, velocity and acceleration of pupillary change, the pupil size for $\mathrm{AD}$ patients is relatively small, and responds slowly.

All participants are elderly, and in addition to being AD patients, their ages may affect pupil responses. Therefore, the effect of two factors (participant group and age level) on pupillary changes is examined using two-way ANOVA. The variables with deviations which contribute most significantly are selected and summarised in Table IV using means across age levels. These means change along with age levels. Most variables selected are related to velocity and time delay. Since there are few significant interactions between the two factors, they may be independent of each other in regards to PLR features. Additionally, most significant differences between age levels appeared for white stimulus and most differences between two groups occurred when w100 light was used. These results may be related to the mechanism of the PLR, and thus a detailed analysis of this will be a topic of our further study.

\section{B. Factor analysis}

There are significant differences in some of the PLR features of the AD and control groups. Though their variables exhibit the qualitative tendencies of a change, their sources could not be determined, as the physical variables and measurement units are completely different; some are expressed using sizes and others are expressed as velocities or accelerations.

Factor analysis was used to extract latent sources of the variables which were measured repeatedly. Since overall integration (int) is a summation of two parts such as int_con and int_rest, it has been omitted during the analysis, thus 14 variables were measured. A two factor structure was estimated using a principal component solution and a screw plot. A factor loading matrix using Promax rotation was produced, as shown in Table V. 
TABLE III

MEANS OF PLR FEATURES

\begin{tabular}{|l|cc|cc|cc|cc|cc|c|}
\hline Feature & \multicolumn{2}{|c|}{ b10(N=19) } & \multicolumn{2}{c|}{ b100(N=17) } & \multicolumn{2}{c|}{ r10(N=18) } & \multicolumn{2}{c|}{ r100(N=19) } & \multicolumn{2}{c|}{ w10(N=19) } & \multicolumn{2}{c|}{ w100(N=19) } \\
Variable & Control & Patient & Control & Patient & Control & Patient & Control & Patient & Control & Patient & Control \\
Patient
\end{tabular}

pairs of bold means show significant differences

TABLE IV

AGE AFFECTED FEATURES (LIST OF SIGNIFICANT VARIABLES)

\begin{tabular}{|l|l|c|c|c|}
\hline \multirow{2}{*}{ Stimulus } & \multirow{2}{*}{ Variable } & \multicolumn{3}{|c|}{ age levels } \\
\cline { 3 - 5 } & b10 & L & M & H \\
\hline b100 & ps_min & -.11 & -.05 & -.04 \\
\hline r10 & t_v_con & 0.0 & 6.9 & 10.9 \\
\hline r100 & t_delay & 0.23 & 0.36 & 0.43 \\
& int_con & 381.4 & 0.24 & 0.31 \\
& RA & 11.2 & 7.9 & 176.3 \\
\hline w10 & v_con & -.64 & -.33 & -.25 \\
& ac_max & -.09 & -.04 & -.03 \\
\hline w100 & RA & 14.3 & 9.6 & 7.6 \\
& v_con & -.64 & -.39 & -.33 \\
& t_v_con & 0.28 & 0.34 & 0.36 \\
& int_con & 459.6 & 280.8 & 217.9 \\
& int_rest & 1279.1 & 853.7 & 659.8 \\
& int & 1738.8 & 1134.5 & 877.7 \\
\hline
\end{tabular}

The fundamental variables of each participant, such as pupil sizes commonly contribute to both factors. The second factor contains variables which are concerned with features of the progress of restoration of the pupil after a pulse of light, and the first factor contains the remaining variables of the features of PLR. As mentioned above, both factors contain two variables, and there is a significant correlation between these two factors $(r=0.38)$. Since even the contribution ratio of each factor when the other factors are eliminated is over $60 \%$, the two factors can account for the deviation.

The factor scores (factor 1 , factor 2 ) were calculated using the factor loading matrix, and their means for each stimuli are summarised in Figure 3 according to group. The horizontal axis indicates the first factor, and the vertical axis indicates the second factor. The error bars show standard errors. The two groups are indicated using suffixes, such as "p" for patients, or "c" for the control group.

When means between the two groups are compared for red (r10 and r100) or white (w10 and w100) stimulus lights, they
TABLE V

FACTOR LOADING MATRIX FOR PLR FEATURES WITH PROMAX ROTATION

\begin{tabular}{|l|c|c|}
\hline Variables & Factor1 & Factor2 \\
\hline ps_base & $\mathbf{0 . 5 9 6}$ & $\mathbf{0 . 5 8 6}$ \\
ps_lon & $\mathbf{0 . 5 9 6}$ & $\mathbf{0 . 5 8 6}$ \\
\hline int_rest & $\mathbf{1 . 0 0 7}$ & -0.271 \\
RA & $\mathbf{0 . 9 9 7}$ & -0.064 \\
int_con & $\mathbf{0 . 9 8 8}$ & 0.006 \\
v_con & $\mathbf{- 0 . 9 0 6}$ & -0.067 \\
ac_max & $\mathbf{- 0 . 9 0 2}$ & -0.021 \\
t_v_con & $\mathbf{- 0 . 7 3 7}$ & 0.110 \\
t_delay & $\mathbf{- 0 . 7 1 2}$ & 0.062 \\
v_rest & $\mathbf{0 . 6 4 3}$ & 0.099 \\
\hline ps_min & -0.111 & $\mathbf{1 . 0 2 5}$ \\
ps_loff & -0.079 & $\mathbf{0 . 9 9 9}$ \\
t_min & 0.086 & $\mathbf{- 0 . 6 4 3}$ \\
t_v_rest & 0.066 & $\mathbf{- 0 . 4 3 8}$ \\
\hline \hline Contribution ratio(1) & 0.42 & 0.21 \\
Contribution ratio(2) & 0.52 & 0.32 \\
\hline \multicolumn{2}{|c|}{ Correlation between factors } \\
\hline \multicolumn{2}{|c|}{ (1): Each factor with other factors eliminated }
\end{tabular}

(2): Each factor with other factors ignored

are located in proximity to each other, but the means of the two groups are relatively far apart from each other for blue stimulus lights (b10 and b100). Pupil reactions in response to light colours are represented in Figure 3. In the results of applying t-tests to pairs of the means for the two subject groups, there are significant differences in the first factor scores for b100 $(t(15)=2.64, p<0.05)$, in the second factor scores for b100 $(t(15)=2.88, p<0.05)$ and for $w 100(t(17)=2.15$, $p<0.05)$. Also, differences without much significance were observed for the second factor scores for b10 $(t(17)=2.07$, $p<0.10)$ and for $\mathrm{r} 10(t(17)=1.77, p<0.10)$.

The results show that between the two subject groups there are significant differences in both factor scores for the b100 condition. As Table III shows many significant differences, the factor scores also represent these differences. Also, the second factor seems to reflect the difference in features of PLR between the two groups. 


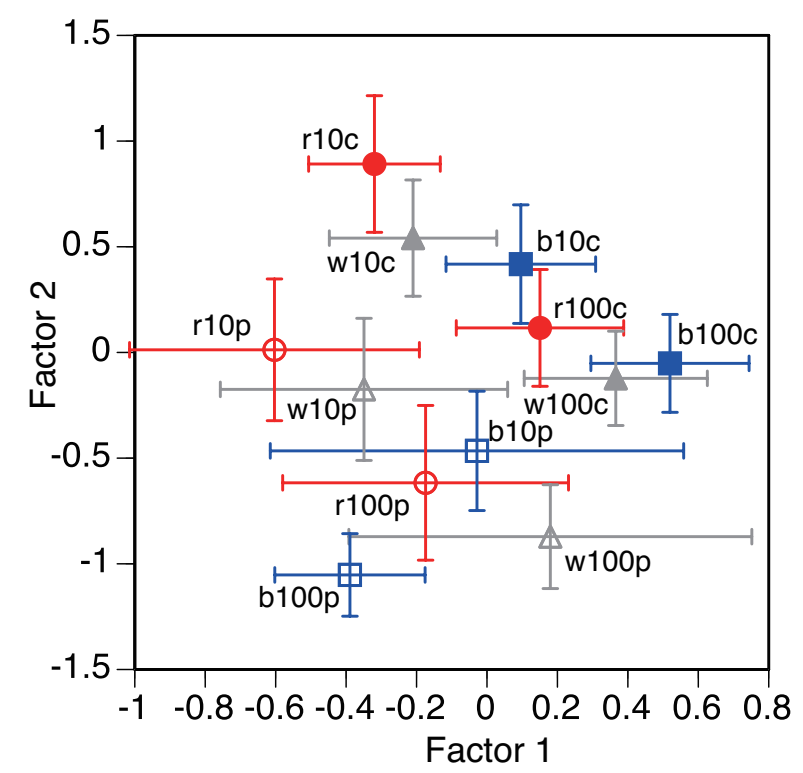

Fig. 3. Distributions of factor scores with error bars showing standard errors

TABLE VI

PERFORMANCE OF MODELS

\begin{tabular}{|l|c|c|c|c|}
\hline Stimulus & AIC & $R^{2}$ & Accuracy & AUC \\
\hline b10 & 25.16 & 0.26 & 82.1 & 0.82 \\
b100 & 17.24 & 0.47 & 89.4 & 0.89 \\
r10 & 27.33 & 0.17 & 73.8 & 0.74 \\
r100 & 28.05 & 0.14 & 72.6 & 0.73 \\
w10 & 27.35 & 0.18 & 69.0 & 0.69 \\
w100 & 24.70 & 0.28 & 79.8 & 0.80 \\
\hline b(10+100) & 21.07 & 0.48 & 89.4 & 0.89 \\
r(10+100) & 30.26 & 0.22 & 71.4 & 0.71 \\
w(10+100) & 22.94 & 0.47 & 90.5 & 0.91 \\
\hline b+r & 18.50 & 0.73 & 100 & 1.00 \\
r+w & 29.70 & 0.50 & 95.2 & 0.95 \\
b+w & 18.00 & 0.73 & 100 & 1.00 \\
\hline b+r+w & 26.00 & 0.73 & 100 & 1.00 \\
\hline
\end{tabular}

Though the influence of age level on factor scores was analysed, it did not affect either factor.

\section{Introducing logistic regression}

As mentioned in the introduction, this paper introduces logistic regression analysis to estimate the probability of diagnosing AD patients using a binary response variable $(p)$ and PLR features. Here, $p=1$ for the control subject and $p=0$ for the AD patient, then $p$ is given by the following equation with logit function.

$$
\begin{array}{r}
\hat{y}_{i}=a+b_{1} \text { factor } 1_{i, j}+b_{2} \text { factor } 2_{i, j} \\
p_{i}=\operatorname{logit}^{-1}\left(\hat{y}_{i}\right)=\frac{1}{1+\exp \left(-\hat{y}_{i}\right)}
\end{array}
$$

Suffix $i$ represents the subject, and suffix $j$ represents the stimulus light condition.

Logistic regression analysis was applied to the above factor scores for several conditions, such as the two factor scores for a specific stimulus light condition (light intensity: 10 or 100),

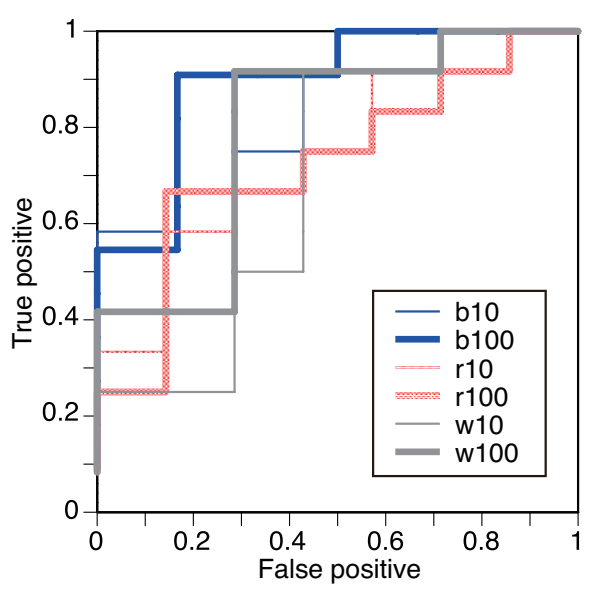

Fig. 4. Comparison of ROC curves

four factor scores for a colour condition (blue, red and white including light intensity 10 and 100) and further combinations such as two colours or all conditions ( 6 conditions and 2 factor scores). Every model was evaluated for fitness of model using AIC (Akaike Information Criteria), and prediction accuracy using $R^{2}$. The accuracy was measured using an appropriate threshold, and performance was summarised using two dimensional metrics such as true positive and false positive. The relationships are then illustrated as ROC (Receiver Operating Characteristics) curves. Figure 4 shows ROC curves of every stimulus condition. The surface area of the curve is also a measure of the AUC (the area under the ROC curve). Their indices are summarised in Table VI.

Results of analyses suggest that discriminant performance is higher for blue light stimuli, in particular for the b100 condition. The ROC curves show step-wise changes, since the number of participants influenced the results. However, the performance of AUC for b100 produced the highest reaction of any single stimulus condition.

\section{Model parameter estimation}

As Table III shows the possibility of discriminating between $\mathrm{AD}$ patients and control group subjects using a logistic regression function. However, the parameters of these functions can not be estimated sufficiently because the amount of data is too limited. Here, the Markov chain Monte Carlo (MCMC) method was introduced for more accurate estimation of the parameters. In regards to the data generation procedure using the MCMC technique, the burn-in period was 2000 and the number of samples was 10000 [12]. Using this procedure for the b100 condition, the parameters are estimated as follows.

$$
\hat{y}_{i}=5.4229+0.4722 * \text { factor } 1_{i}+7.3801 * \text { factor } 2_{i}
$$

The magnitude of coefficient for the second factor ( factor 2 ) is 15 times that of the coefficient for the first factor ( factor 1 ).

As an additional example, the parameters for blue light stimuli were estimated as follows: 


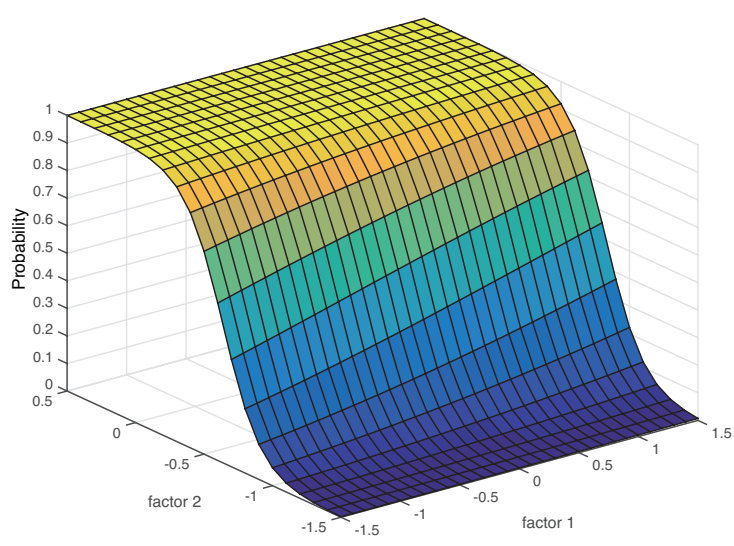

Fig. 5. Probability distribution for b100

$$
\begin{array}{r}
\hat{y}_{i}=9.1664+2.5320 * \text { factor } 1_{i, b 10}-1.1366 * \text { factor } 2_{i, b 10} \\
-1.4011 * \text { factor } 1_{i, b 100}+12.0891 * \text { factor } 2_{i, b 100}
\end{array}
$$

The magnitude of these parameters suggests that the coefficient for the second factor at a high level of brightness (b100) is relatively larger than for others (b10 and the first factor at a low level of brightness). As the patterns of coefficients depend on the light stimulus, the wavelengths of the stimuli may affect these reactions.

In regards to the discussion in the previous section, the probability of control group subjects (1) or AD patients (0) may be illustrated using two dimensional information (factor 1 and factor 2), as shown in Figure 5. Figure 5 shows that the probability distribution against patients with $\mathrm{AD}$ depends mainly on the scores of the second factor. Also, the score of the first factor helps to more finely adjust the probability during the period where the curve is steep.

In regards to the experimental procedure, the features of PLR can be measured best during a one second high brightness level pulse of blue light (b100). Also, factor scores were calculated using the factor loading matrix shown in Table V. Finally, the probability of diagnosing AD patients can be predicted using the function above.

However, the possibility of developing a more flexible procedure for use in future experiments involving additional new participants will be a subject of our further study.

\section{SUMmary}

This paper presents a procedure for predicting the probability of diagnosing AD patients using features of PLR, which respond to the activities of ipRGCs.

Three colour lights at two levels of brightness were illuminated for 1 second, and pupil light responses were observed. 15 features were extracted from each PLR, and two factor scores were calculated using a factor loading matrix. The following results were produced.
1) There are significant differences in some features between $\mathrm{AD}$ patients and control group subjects, in particular for the b100 condition. Also, for a few features for white light there are significant differences between age levels.

2) Logistic regression analysis was introduced to discriminate $\mathrm{AD}$ patients from the control group using two factor scores in response to chromatic stimuli. The performance was evaluated using the indices of the fitness of equations. As a result, the performance for b100 was the highest.

3) The MCMC technique was introduced to estimate the parameters of the regression functions. The model provides a distribution of probability for $\mathrm{AD}$ patients and the control group.

The validity of the probability estimations should be confirmed using the PLR data of patients. This will be a subject of our further study.

\section{ACKNOWLEDGMENT}

Polish Ministry of Science and Higher Education research grant NN518 405338 partially supported this research.

\section{REFERENCES}

[1] D. F. Fotiou, V. Setergiou, D. Tsiptsios, C. Lithari, M. Nakou, and A. Karlovasitou, "Cholinergic deficiency in Alzheimer's and Parkinson's disease: Evaluation with pupillometry," International Journal of Psychophysiology, vol. 73, pp. 143-149, 2009.

[2] D. M. Bittner, I. Wieseler, H. Wilhelm, M. W. Riepe, and N. G. Müller, "Repetitive pupil light reflex: Potential marker in Alzheimer's disease?" Journal of Alzheimer's Disease, vol. 42, pp. 1469-1477, 2014.

[3] P. D. Gamlin, D. H. McDougal, and J. Pokorny, "Human and macaque pupil responses driven by melanopisn-containing retinal ganglion cells," Vision Research, vol. 47, pp. 946-954, 2007.

[4] A. Kawasaki and R. H. Kardon, "Intrinsically photosensitive retinal ganglion cells," Journal of Neuro-Ophthalmology, vol. 27, pp. 195-204, 2007.

[5] M. Nakayama, W. Nowak, H. Ishikawa, K. Asakawa, and Y. Ichibe, "Discovering irregular pupil light responses to chromatic stimuli using waveform shapes of pupillograms," EURASIP J. in Bioinformatics and System Biology, no. \#18, pp. 1-14, 2014.

[6] T. Yoshida, K. Ohno-Matsui, S. Ichinose, T. Sato, N. Iwasa, T. C. Saido, T. Hisatomi, M. Mochizuki, and I. Morita, "The potential role of amyloid $\beta$ in the pathogenesis of age-related macular degeneration," The Journal of Clinical Investigation, vol. 115, no. 10, pp. 2793-2800, 2005.

[7] J.-D. Ding, J. Lin, B. Mace, R. Herrmann, P. Sullivin, and C. Rickman, "Targeting age-related macular degeneration with alzheimer's disease based immunotherapies: Anti-amyloid- $\beta$ antibody attenuates pathologies in an age-related macular degeneration mouse model," Vision Research, vol. 48, pp. 339-345, 2008

[8] K. Ohno-Matsui, "Parallel findings in age-related macular degeneration and alzheimer's disease," Progress in Retinal and Eye Research, vol. 30 , pp. 217-238, 2011.

[9] J. M. Sivak, "The aging eye: Common degenerative mechanisms between the alzheimer's brain and retinal disease," Investigative Ophthalmology \& Visual Science, vol. 54, no. 1, pp. 871-880, 2013.

[10] W. Nowak, M. Nakayama, M. Pieniążek, and A. Hachoł, "Feature analyses of pupil light reflex to chromatic stimuli in alzheimer's patients," in Proceedings of 2nd International Conference on Frontiers of Signal Processing, 2016, pp. 58-62.

[11] W. Nowak, A. Zarowska, E. Szul-Pietrzak, and M. Misiuk-Hojło, "System and measurement method for binocular pupillometry to study pupil size variability," BioMedical Engineering Online, vol. 13, no. \#69, pp. 1-16, 2014.

[12] Sas/stat 13.1 user's guide, the $\mathrm{mcmc}$ procedure. [Online]. Available: http://support.sas.com/documentation/onlinedoc/stat/131/mcmc.pdf 